\title{
Plasma Polymerized Films for Mass Sensitive Biosensors
}

\author{
Gizem Kaleli Can ${ }^{*}$ (D), Selma Mutlu 2 (D), Mehmet Mutlu ${ }^{3}$ (iD)
}

\section{INTRODUCTION}

The development of the microprocessor technology and biotechnology have affected the growth rate of biosensor technology [1-2]. Biosensor technology can be used in different areas like biomedical applications, food, environmental, military and etc [2-6]. Considering biomedical applications in particular, target analytes that are indicators of a disease, biological and chemical contaminants or disease-causing micro-organisms should be determined for disease monitoring [7]. Conventional methods used during the detection of these markers have some drawbacks arisen from a series of time-consuming procedure requirement and the usage of complex devices including separative techniques coupled to various detectors such as GC, HPLC, MS, UV and etc. when compared with biosensors [6,8-9]. Additionally, trained personnel are needed to operate time-consuming and complex conventional systems. Due to the demand for rapid, sensitive, selective and accurate methods to detect these target analytes, the development of biosensors became crucial [10].

In biosensors, there are five different transduction system which is known as electrochemical, electrical, piezoelectric (mass-sensitive), thermometric (calorimetric) or optical biosensors [11]. Among these types of biosensors, mass sensitive biosensors are used in many studies due to their high sensitivity, selectivity, label-free operation and low cost [6]. Quartz crystal microbalance $(\mathrm{QCM})$ is the most preferred type of crystal used in mass-sensitive biosensors.

The working principle of QCM can be summarized that the damping in oscillation frequency is proportional with the adsorption of material on the surface of the crystals. It is reported that when an $9 \mathrm{MHz}$ of AT-cut quartz crystal is used as a crystal, the adsorption of mass about $1 \mathrm{ng}$ changes frequency shift of $1 \mathrm{~Hz}$ [12-13]. In recent years, the quartz tuning fork (QTF), which has the same working principle as QCM, has shown that it can be an alternative to QCM by drawing attention to its high sensitivity due to its high-quality factor (QF 10000-7000), high frequency stability, sharp frequency response, repeatability in measurements, convenience for mass detection and low cost. These features of QTF, both quartz and fork-like geometry, allow it to pass in front of silicon cantilever and QCM as an inverter. To obtain mass sensitive sensor with good performance for both 
crystals, a properly designed biosensor is essential [14].

Surface modification of piezoelectric crystal is crucial step to turn crystals sensing platform to bio-sensing. Many polymeric layers such as cellulose acetate, polyurethane, polystyrene, polyethylene amine, polybutyl methacrylate, polyvinyl formal/ethylal, polyvinylalcohol, and polyethyleneimine have been used for the creation of interface for immobilization of bioreceptor [15-22]. Additionally, many coating methods like silanization, immobilization on the pre-coated crystal, entrapment, or cross-linking methods are used to immobilize bioreceptor such as antibody, aptamer and nucleic acids onto the quartz crystal [6, 16, 18-20, 22-24]. However, at the end of these techniques, thicker films are obtained on the crystals' surfaces which diminishes performance by mass overload, and create a harsh environment, which reduces biological activity.

Nowadays, plasma technologies have been started to be used for the modification of the surface of the quartz crystal [14, 19, 25-32]. This technique give chance to cover the crystal surfaces with control film growth down to the angstrom (Å) scale with uniform coating, while preserving the bulk properties of the substrate material. The obtained layer enhances QCM sensitivity and the immobilization capacity of bioactive agents by obtaining functional groups on recognition layers [22, 28, 33].

In this review, the principle of detection, surface modification of piezoelectric crystals by plasma technology and applications of these crystals as a mass sensitive biosensor in biomedical applications are summarized in concept of the theory and the literature in this field.

\section{Working Principle of Mass Sensitive Biosensors}

In both QCM and QTF biosensors, biological component is quantified by measuring the frequency change, which corresponds to a mass change of the sensor surface. In this sensor type, no potentially hazardous-labeled materials are needed to be used [34].

\subsection{Detection Scheme of Quartz Crystal Microbalance}

Quartz crystal placed in QCM device as a transducer. The preparation of this transducer is achieved by sandwiching of a quartz crystal wafer between two metal electrodes like gold, silver, aluminum, or nickel [35]. Then, an external oscillator circuit are connected to the electrodes and this circuit drives the quartz crystal at its resonant frequency. The resonant frequency of the crystal is directly based on the properties of crystal like the cutting edge, thickness and etc. The quartz plates are used as a biosensor are mostly 5, 9, or $10 \mathrm{MHz}$ AT-cut quartz crystal.

The detection of biological molecule by quartz crystal microbalance (QCM) depends on the measurement of the mass changes and physical properties of thin layers deposited on the crystal surfaces [36-38]. Therefore, these highly precise and stable quartz crystal is a good transducer to be used as a biosensor by monitoring its frequency changes.

Sauerbrey described the working principle of the sensor for the gaseous phase by the relation between the mass loading on quartz crystals and the corresponding change in resonant frequency of the crystal [39]:

$\Delta \mathrm{f}=-2 f_{0}^{2} \Delta \mathrm{m} / \mathrm{A}\left(\rho_{q} \mu_{q}\right)^{1 / 2}$

where $\Delta \mathrm{f}$ is the frequency change of the crystal resonance, $f_{0}$ is the fundamental frequency of the crystal (in $\mathrm{Hz}$ ), $\mathrm{A}$ is the surface area (in $\mathrm{cm}^{2}$ ), $\Delta \mathrm{m}$ is the deposited mass (in $\mathrm{g}$ ), $\rho_{q}$ and $\mu_{q}$ is the properties of the density and the shear modulus of crystal, respectively.

By inserting the values of properties (density, $\rho_{q}=2.648 \mathrm{~g} \mathrm{~cm}^{-3}$; shear modulus, $\mu_{q}=2.987 \times 1011 \mathrm{~g} \mathrm{~cm}^{-1} \mathrm{~s}^{-2}$ ) for an AT cut crystal, the above relationship can be rearranged as:

$\Delta \mathrm{f}=-2.27 \times 10^{-6} f_{0}^{2} \Delta \mathrm{m} / \mathrm{A}$

The Sauerbrey equation gives the frequency shift measured in air corresponding the coated materials. The equation is modified for the frequency measurement in liquid phase due to bulk liquid properties like conductivity, viscosity, density and dielectric constant. In the liquid phase, the frequency shifts are determined by the Bruckenstein-Shay and Kanazawa-Gordon's equation as follows [31-32]:

$\Delta \mathrm{f}=-f_{0}^{3 / 2}\left(\rho_{L} \eta_{L} / \pi \rho_{q} \mu_{q}\right)^{1 / 2}$

where $\eta_{L}$ and $\rho_{L}$ are the absolute viscosity and density of the liquid, respectively [6].

\subsection{Detection Scheme of Quartz Tuning Fork}

Quartz tuning fork has become a widely used component in frequency measurement due to their high stability, quality factor, accuracy and low power consumption. These forks have replaced the mechanical pendulum and spring used in watches since the late 1960s, allowing the production of more stable watches. The key component of these high-stability watches, QTF, is manufactured by mass production at low cost, making QTF use even more attractive [42-51].

Nowadays, QTFs have been started to be used as an alternative to microfabricated silicone in atomic force microscopy cantilevers. However, QTF is one step ahead due to their quartz material and their fork-shaped geometry compared to silicone cantilevers. The reason for this is explained by the fact that mechanical movement in QTF with two prongs, reducing the damping and dramatic decrement of high-quality factor even in the air. 
Basically, the QTF consists of two forks which is coated with metal films. These forks vibrate under vacuum at a frequency of $32758 \mathrm{~Hz}$, moving lateral when stimulated by alternating current excitation voltage [14, 42-51]. When hermetic casing of the QTFs were removed, resonance frequency of QTFs decreased due to air damping. This change not only affects the frequency but also changes the quality factor $\left(Q_{\text {vacuum }} \leqslant 100,000\right.$ and $\left.Q_{\text {air }} \leqslant 10,000\right)$.

As mentioned, the working principle of QTF is similar to that of QCM. However, there are very few studies on its use in biosensors. The first and last use of QTF as a biosensor was performed by Su et al. (2002). In this study, anti-IgG was detected on polystyrene modified surfaces and the responses of the decorated surfaces against immunoglobulin $\mathrm{G}(\mathrm{IgG})$ were tested. As a result, it was found that the decorated QTF could work in the linear range of 5-100 $\mu \mathrm{g} \mathrm{ml} \mathrm{l}^{-1}$, but its reproducibility was low $(\% \mathrm{CV}=11 \%-16 \%)$. The reason of low reproducibility could be the nonhomogeneous polystyrene coating [52].

\section{Surface Modification of Piezoelectric Crystals}

Beside all advantages of both crystals in biosensor application, performance, reliability and stability of crystals have been directly affected from surface modification steps required for the functionalization and activation of the QCM and QTF surface, prior to bioreceptor immobilization. Therefore, to obtain highly sensitive and selective mass-sensitive biosensors for target analyte detection, surface modification of the QCM and QTF to create a recognition layer is crucial.

Plasma treatments can advantageously replace thermal, radiative, or chemical processes for the surface modification of materials. In fact, these treatments can be easily controlled and are environmentally friendly. Moreover, plasma modifies the surface layer at a depth from 50 to $500 \AA$, depending on power and time, leaves the bulk characteristic unaffected.

Plasma polymerized films are pinhole-free and highly cross-linked and therefore are insoluble, thermally stable, chemically inert and mechanically tough. Furthermore, such films are often highly coherent and adherent to a variety of substrates including conventional polymer, glass and metal surfaces. Depending upon the precursor that has been employed in plasma processing, the surface energy would be increased many times or the chemical structure could be converted into branched molecule groups to immobilize biological components such as antibody, enzyme, protein and etc.

\subsection{Surface Modification of Piezoelectric Crystals by Plasma Technology}

Fourth state of matter, plasma, is composed of excited atoms, molecules, ions and radicals [53-54]. Plasma state occurs with the help of electrons generated by radio frequency (RF), microwave or hot filament discharge. This is a chaotic reactive chemical medium in which many plasma-surface reactions take place. Intense amounts of ions and excited species in the plasma can change the surface properties of even an inert material, ceramic [14, 53-54]. In addition, plasma techniques can successfully modify many complex materials $[14,53-55]$.

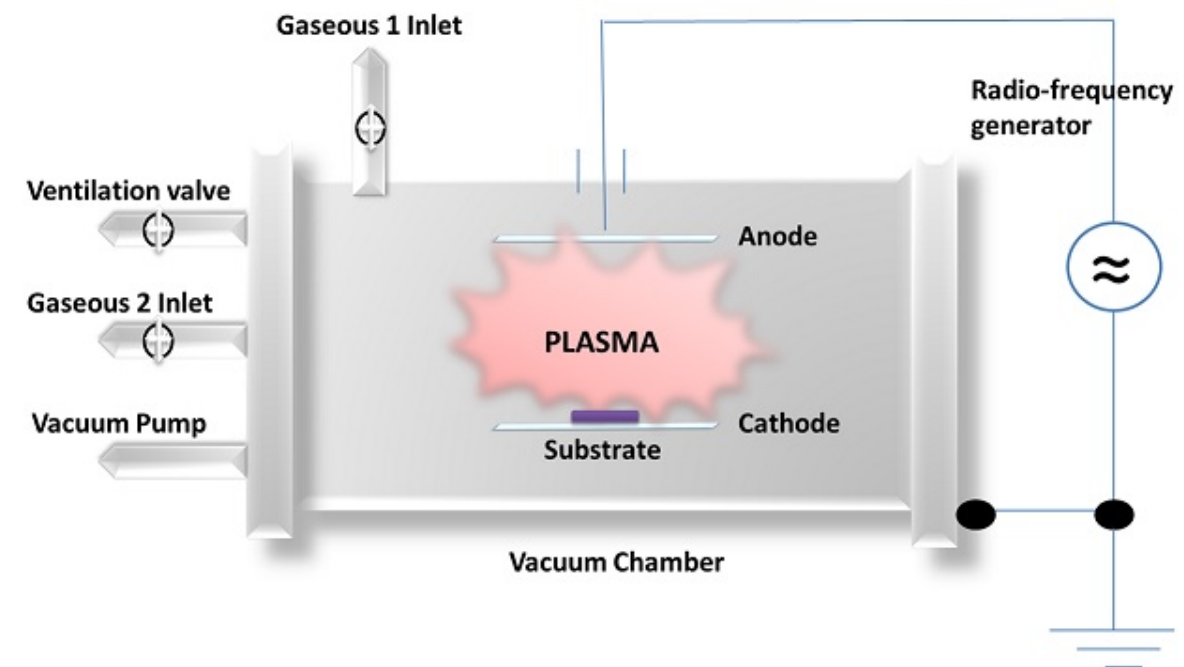

Figure 1. Schematic representation of the radio-frequency generated low-pressure plasma system.

Plasma techniques can make many changes such as changing the surface energy of the material, increasing the adhesion strength and biocompatibility. In addition, as a result of plasma modification, inexpensive thin films can be produced independent from the geometry of the material and structure of the material (metal, polymer, ceramic and / or composite). The most important advantage of this technique is that it is possible to change the chemical, electrical, optical, mechanical and biological structure of the material surface with high efficiency without affecting the bulk structure. This technique can be also used in the microelectronics industry for surface sterilization and cleaning, modification of the surface with a specific pattern in the case of masking [14, 53-54]. 
After plasma phase is formed with plasma technology, various changes can be made on the surface by plasma spraying, etching, cleaning, implantation and deposition [53]. Among them, plasma deposition has been mostly used in surface modification of mass sensitive biosensor. Plasma deposition method is preferred in many areas because the obtained coating may show completely different properties from the bulk. Inductively coupled plasma deposition, plasma-graft co-polymerization and plasma polymerization are techniques used to perform this method [14, 53-54]

In plasma polymerization method, polymer synthesis from monomer is achieved with the help of energetic electrons, ions and radicals found in chaotic plasma medium. Plasma polymerization is different compared to conventional polymerization methods because of the existence of both ions and radicals. Generally, the chemical composition of polymeric thin film produced by plasma polymerization differs from the polymer prepared by the conventional radical or ionic polymerization reaction. This difference is caused by the polymer formation mechanism. Plasma polymerization with plasma includes some steps like the formation of radicals from monomer by plasma activation, recombination of obtained radicals, and reactivation of recombinant molecules. Unlike the polymer synthesized by conventional polymerization reactions, the plasma polymer consists of repeating monomer units, highly cross-linked, fragmented and reordered complex units [14, 53-54].

\section{Applications of plasma modified QCM and QTF in Biomedical Applications}

In the literature, plasma modified mass sensitive biosensors used in biomedical application have been presented for detecting genetically modified organism, human pathogen and mostly model protein, bovine serum albumin (BSA) over the past decade [14, 19, 29-32]. A DNA biosensor, which uses single-stranded DNA probe to detect human pathogen, Vibrio parahaemolyticus, based on a quartz crystal microbalance has been used. This single-stranded DNA probe was immobilized to QCM's surface by the help of functional groups formed by plasma polymerization of hexamethyldisilazane. After surface modification using plasma deposition, sensitivity of QCM increased to $86 \mathrm{ng} / \mathrm{ml}$ and linear range was found $86-468 \mathrm{ng} / \mathrm{mL}$ [29]. The other research was about the producing DNA biosensor to detect CaMV 35S promoter sequence (P35S) which is commonly inserted in the genome of the GMO regulating the transgene expression and the performance of the biosensor was evaluated with genomic DNAs of pflp gene-inserted transgenic tobacco plants. The immobilization was achieved with two different ways. These are chemisorption of thiolated probe on gold through thiol-gold interaction and covalent attachment of amines probe through glutaraldehyde activation. Amine probes were produced by ethylenediamine using plasma polymerization to produce amine probes on the quartz crystal surface. However, amine probes were not found efficient [30]. A study describing a new strategy to increase the performance of mass sensitive biosensor via electrospinning and plasma polymerization techniques was reported. Quartz crystal surface was firstly coated with polyvinyl alcohol nanofibers to increase surface to volume ratio. Then, nanofibers located on QCM were modified with allylamine monomer using plasma polymerization technique. The performance of modified QCM was tested with model protein, BSA and $548 \pm 4 \mathrm{~Hz}$ and $50 \pm 5 \mathrm{~Hz}$ frequency shifts were obtained for "dip and dry" method and "flow-cell method", respectively [19]. Our research group conducted a novel research to understand the effect of plasma-polymerized amine-rich thin films over QTF surfaces and to test their usability as a mass sensitive biosensor. Precursors, amylamine (amy), n-heptylamine (hep), or diaminocyclohexane (dach), were selected as a monomer for plasma polymerization technique and the performance of plasma modified QTF was studied by detecting model protein, BSA. According to detachment performance of thin films during glutaraldehyde activation, surfaces that produced by using amylamine was found optimum. After that, amylamine functionalized QTFs were tested with BSA $(100 \mu \mathrm{g} / \mathrm{mL})$ and resonance frequencies were decreased about $5 \mathrm{~Hz}$ [31]. At last, a QTF-based platform was produced by using a bi-layer film that coated by plasma-polymerized n-heptane (hep) and then by ethylenediamine (EDA), respectively in our laboratory. Then, the BSA detection performance of mass sensitive biosensor was tested and $20 \pm 3.60 \mathrm{~Hz}$ [32].

\section{Future Trends}

In this chapter, biomedical application by plasma modified QCM and QTF-based mass sensitive biosensor is briefly summarized. Researches shown that plasma polymerization can be used for surface modification of piezoelectric crystals. The analysis with plasma polymerized crystals generally focused on the model protein, BSA, and etc. The plasma modified piezoelectric biosensors appear to be a suitable and convenient tool for detection of biomarkers. By virtue of this approach, it seems that both QCM and QTF will be favorable tool as a diagnosing disease. Future investigations can be focus on making systematic comparison between the performance of plasma modified-QCM and that of plasma modified-QTF. 


\section{REFERENCES}

[1] Taleat, Z., Khoshroo, A., Mazloum-Ardakani, M. (2014). Screen-printed electrodes for biosensing: a review (2008-2013). Microchimica Acta, 181(9-10), 865-891.

[2] Goode, J. A., Rushworth, J. V. H., Millner, P. A. (2014). Biosensor regeneration: a review of common techniques and outcomes. Langmuir, 31(23), 6267-6276.

[3] Wang, J. (2006). Electrochemical biosensors: towards point-of-care cancer diagnostics. Biosensors and Bioelectronics, 21(10), 1887-1892.

[4] Huet, A. C., Delahaut, P., Fodey, T., Haughey, S. A., Elliott, C., \& Weigel, S. (2010). Advances in biosensor-based analysis for antimicrobial residues in foods. TrAC Trends in Analytical Chemistry, 29(11), 1281-1294.

[5] Weller, M. G., Schuetz, A. J., Winklmair, M., \& Niessner, R. (1999). Highly parallel affinity sensor for the detection of environmental contaminants in water. Analytica chimica acta, 393(1-3), 29-41.

[6] Mutlu, S., (2011). Mass Sensitive Biosensors: Principles and Applications in Food in: Mutlu M. (Ed.), Biosensors in Food Processing, Safety, and Quality Control. Taylor and Francis, CRC Press, New York, pp. 71-89.

[7] Andreescu, S., Sadik, O. A. (2004). Trends and challenges in biochemical sensors for clinical and environmental monitoring. Pure and applied chemistry, 76(4), 861-878.

[8] Alocilja, E. C. and Radke, S. M. (2003). Market analysis of biosensors for food safety. Biosensors and Bioelectronics, $18(5-6), 841-846$.

[9] Öztürk, K., Durusoy, M., Pişkin, E., 2008. Journal of Bioavtive and Compatible Polymers. 26(6), 579-593.

[10] Ricci, F., Volpe, G., Micheli, L., Palleschi, G. (2007). A review on novel developments and applications of immunosensors in food analysis. Analytica chimica acta, 605(2), 111-129.

[11] Monošík, R., Stred'anský, M., Šturdík, E. (2012). Biosensors-classification, characterization and new trends. Acta Chimica Slovaca, 5(1), 109-120.

[12] Kurosawa, S., Tawara, E., Kamo, N., Kobatake, Y. (1990). Oscillating frequency of piezoelectric quartz crystal in solutions. Analytica Chimica Acta, 230, 41-49.

[13] Muratsugu, M., Ohta, F., Miya, Y., Hosokawa, T., Kurosawa, S., Kamo, N., Ikeda, H. (1993). Quartz crystal microbalance for the detection of microgram quantities of human serum albumin: relationship between the frequency change and the mass of protein adsorbed. Analytical chemistry, 65(20), 2933-2937.

[14] Kaleli Can, G., Development of QTF-Based Mass Sensitive Immunosensor for Phenylketonuria Diagnosis. (TOBB University of Economics and Technology, ANKARA, 2018).

[15] Alp, B., Mutlu, S., Mutlu, M. (2000). Glow-discharge-treated cellulose acetate (CA) membrane for a high linearity single-layer glucose electrode in the food industry. Food Research International, 33(2), 107-112.

[16] Jenik, M., Seifner, A., Lieberzeit, P., Dickert, F. L. (2009). Pollen-imprinted polyurethanes for QCM allergen sensors. Analytical and bioanalytical chemistry, 394(2), 523-528.

[17] Koshets, I. A., Kazantseva, Z. I., Shirshov, Y. M. (2003). Polymer films as sensitive coatings for quartz crystal microbalance sensors array. Semiconductor Physics Quantum Electronics \& Optoelectronics.

[18] Öztürk, K., Durusoy, M., Pişkin, E. (2008). A simple quartz crystal microbalance nucleic acid sensor for detection of telomerase. Journal of Bioactive and Compatible Polymers, 23(6), 579-593. 
[19] Rodoplu, D., Sen, Y., Mutlu, M. (2013). Modification of quartz crystal microbalance surfaces via electrospun nanofibers intended for biosensor applications. Nanoscience and Nanotechnology Letters, 5(4), 444-451.

[20] Tsai, W. B., Chien, C. Y., Thissen, H., Lai, J. Y. (2011). Dopamine-assisted immobilization of poly (ethylene imine) based polymers for control of cell-surface interactions. Acta biomaterialia, 7(6), 2518-2525.

[21] Zhang, C., Cappleman, B. P., Defibaugh-Chavez, M., Weinkauf, D. H. (2003). Glassy polymer-sorption phenomena measured with a quartz crystal microbalance technique. Journal of Polymer Science Part B: Polymer Physics, 41(18), 2109-2118.

[22] Kabay, G., Can, G. K., \& Mutlu, M. (2017). Amyloid-like protein nanofibrous membranes as a sensing layer infrastructure for the design of mass-sensitive biosensors. Biosensors and Bioelectronics, 97, 285-291.

[23] Park, I. S., Kim, N. (1998). Thiolated Salmonella antibody immobilization onto the gold surface of piezoelectric quartz crystal. Biosensors and Bioelectronics, 13(10), 1091-1097.

[24] Park, I. S., Kim, D. K., Adanyi, N., Varadi, M., Kim, N. (2004). Development of a direct-binding chloramphenicol sensor based on thiol or sulfide mediated self-assembled antibody monolayers. Biosensors and Bioelectronics, 19(7), 667-674.

[25] Nakanishi, K., Muguruma, H., \& Karube, I. (1996). A novel method of immobilizing antibodies on a quartz crystal microbalance using plasma-polymerized films for immunosensors. Analytical chemistry, 68(10), 1695-1700.

[26] Mutlu, S., Saber, R., Koçum, C., Piskin, E. (1999). An immunosensor: Immobilization of anti-HBs antibody on glow-discharge treated piezoelectric quartz crystal for HBs-AG detection. Analytical Letters, 32(2), 317-334.

[27] Wu, Z., Yan, Y., Shen, G., Yu, R. (2000). A novel approach of antibody immobilization based on n-butyl amine plasma-polymerized films for immunosensors. Analytica chimica acta, 412(1-2), 29-35.

[28] JMutlu, S., Çökeliler, D., Shard, A., Goktas, H., Ozansoy, B., Mutlu, M. (2008). Preparation and characterization of ethylenediamine and cysteamine plasma polymerized films on piezoelectric quartz crystal surfaces for a biosensor. Thin Solid Films, 516(6), 1249-1255.

[29] Chen, K. S., Chen, S. C., Lin, H. R., Yan, T. R., Tseng, C. C. (2007). A novel technique to immobilize DNA on surface of a quartz crystal microbalance by plasma treatment and graft polymerization. Materials Science and Engineering: C, 27(4), 716-724.

[30] Karamollaoğlu, İ., Öktem, H. A., Mutlu, M. (2009). QCM-based DNA biosensor for detection of genetically modified organisms (GMOs). Biochemical Engineering Journal, 44(2-3), 142-150.

[31] Can, G. K., Özgüzar, H. F., Kabay, G., Kömürcü, P., Mutlu, M. (2018). Simultaneous insulation and modification of quartz tuning fork surface by single-step plasma polymerization technique with amine-rich precursors. MRS Communications, 8(2), 541-549.

[32] Özgüzar, H. F., Can, G. K., Kabay, G., Mutlu, M. (2019). Quartz tuning fork as a mass sensitive biosensor platform with a bi-layer film modification via plasma polymerization. MRS Communications, 1-9.

[33] Akdoğan, E., Çökeliler, D., Marcinauskas, L., Valatkevicius, P., Valincius, V., Mutlu, M. (2006). A new method for immunosensor preparation: Atmospheric plasma torch. Surface and Coatings Technology, 201(6), 2540-2546.

[34] Su, X., Chew, F. T., Li, S. F. (2000). Design and application of piezoelectric quartz crystal-based immunoassay. Analytical Sciences, 16(2), 107-114.

[35] Richard, F. T., Jerome, S. S. (1996). Handbook of Chemical and Biochemical Sensors. 
[36] Ballantine Jr, D. S., White, R. M., Martin, S. J., Ricco, A. J., Zellers, E. T., Frye, G. C., Wohltjen, H. (1996). Acoustic wave sensors: theory, design and physico-chemical applications. Elsevier.

[37] Janshoff, A., Galla, H. J., Steinem, C. (2000). Piezoelectric mass-sensing devices as biosensors-an alternative to optical biosensors?. Angewandte Chemie International Edition, 39(22), 4004-4032.

[38] Lu, F., Lee, H. P., Lim, S. P. (2004). Quartz crystal microbalance with rigid mass partially attached on electrode surfaces. Sensors and actuators A: Physical, 112(2-3), 203-210.

[39] Sauerbrey, G. Z. (1959). The use of quarts oscillators for weighing thin layers and for microweighing. Z. Phys., $155,206-222$.

[40] Bruckenstein, S., Shay, M. (1985). Experimental aspects of use of the quartz crystal microbalance in solution. Electrochimica Acta, 30(10), 1295-1300.

[41] CKanazawa, K. K., Gordon, J. G. (1985). Frequency of a quartz microbalance in contact with liquid. Analytical Chemistry, 57(8), 1770-1771.

[42] Castellanos-Gomez, A., Agraït, N., \& Rubio-Bollinger, G. (2010). Carbon fibre tips for scanning probe microscopy based on quartz tuning fork force sensors. Nanotechnology, 21(14), 145702.

[43] Heyde, M., Kulawik, M., Rust, H. P., \& Freund, H. J. (2004). Double quartz tuning fork sensor for low temperature atomic force and scanning tunneling microscopy. Review of scientific instruments, 75(7), 2446-2450.

[44] Labardi, M. (2007). Dynamic force microscopy with quartz tuning forks at high oscillation amplitudes. Nanotechnology, 18(8), 084019.

[45] Song, S. H. (2009). Electrical characterization of a tuning fork crystal oscillator using dual-phase lock-in technique. Review of Scientific Instruments, 80(3), 034703.

[46] Yang, C. H., Chang, T. H., Yang, M. J., Moore, W. J. (2002). A low noise transimpedance amplifier for cryogenically cooled quartz tuning fork force sensors. Review of scientific instruments, 73(7), 2713-2716.

[47] Bettac, A., Koeble, J., Winkler, K., Uder, B., Maier, M., \& Feltz, A. (2009). QPlus: atomic force microscopy on single-crystal insulators with small oscillation amplitudes at 5 K. Nanotechnology, 20(26), 264009.

[48] Giessibl, F. J. (1998). High-speed force sensor for force microscopy and profilometry utilizing a quartz tuning fork. Applied Physics Letters, 73(26), 3956-3958.

[49] Wastl, D. S., Weymouth, A. J., \& Giessibl, F. J. (2013). Optimizing atomic resolution of force microscopy in ambient conditions. Physical Review B, 87(24), 245415.

[50] Chen, Y., Li, Y., Shan, G., Zhang, Y., Wang, Z., Wang, M., Qian, J. (2018). Design and implementation of a novel horizontal AFM probe utilizing a quartz tuning fork. International Journal of Precision Engineering and Manufacturing, 19(1), 39-46.

[51] Friedt, J. M., \& Carry, E. (2007). Introduction to the quartz tuning fork. American Journal of Physics, 75(5), 415422 .

[52] Zhang, J., Su, X. D., \& O'shea, S. J. (2002). Antibody/antigen affinity behavior in liquid environment with electrical impedance analysis of quartz crystal microbalances. Biophysical chemistry, 99(1), 31-41.

[53] Yasuda, H. (1984). Plasma polymerization for protective coatings and composite membranes. Journal of membrane science, 18, 273-284.

[54] Kamińska, A., Kaczmarek, H., \& Kowalonek, J. (2002). The influence of side groups and polarity of polymers on 
the kind and effectiveness of their surface modification by air plasma action. European Polymer Journal, 38(9), 1915-1919.

[55] Mändl, S., \& Rauschenbach, B. (2000). Plasma-Immersions-Ionenimplantation. Ein neues Verfahren zur homogenen Oberflächenmodifizierung komplex geformter medizinischer Implantate-Plasma Immersion Ion Implantation. New Technology for Homogeneous Modification of the Surface of Medical Implants of Complex Shapes. Biomedizinische Technik/Biomedical Engineering, 45(7-8), 193-198. 